\title{
Interdisciplinaridade no ensino superior: um projeto de cooperação Portugal-
}

\author{
Brasil
}

\section{Interdisciplinary practices in higher education: a Portugal-Brazil cooperation}

\section{project}

\author{
Ana Capelo*, Paula Carvalho*, Lucimara Santos**, Luís Menezes*, António Ribeiro*, Anabela Novais*, João Nunes*, \\ Dalila Rodrigues*, Cristina Gomes*, Isabel Abrantes*, Cristiana Mendes*, Helena Gomes*, Ana Patrícia Martins*, \\ Maria João Silva***, Soeli Monte Blanco** \\ *Escola Superior de Educação de Viseu, Instituto Politécnico de Viseu, Portugal; **Centro de Educação à Distância, Universidade \\ do Estado de Santa Catarina, Brasil; ***Escola Superior de Educação de Lisboa, Instituto Politécnico de Lisboa, Portugal
}

\begin{abstract}
Resumen
Como resultado de um protocolo de colaboração internacional entre o Instituto Politécnico de Viseu e a Universidade do Estado de Santa Catarina, o projeto que se apresenta tem por objetivo identificar, caracterizar e partilhar, ao nível de Instituições de Ensino Superior (IES), iniciativas desenvolvidas nos cursos de formação inicial de professores que potenciem a promoção de competências de planificação, implementação e avaliação de práticas interdisciplinares (PIs), incluindo as que incentivem ligações com contextos formais/não formais. Em termos metodológicos, compreende três fases (clarificação, monitorização e divulgação) e baseia-se numa metodologia mista, com pendor qualitativo. Espera-se que o conhecimento produzido sirva de base ao design de PIs. Palavras-chave: Interdisciplinaridade; práticas na formação de professores; ensino superior; cooperação internacional
\end{abstract}

\section{Abstract}

This project is the result of an international collaboration protocol between the Polytechnical Institute of Viseu (Portugal) and the University of Santa Catarina State (Brasil). At the level of higher education institutions (HEIs), it aims to identify, characterize and share initiatives in teacher training courses which promote the skills of planning, implementation and evaluation of interdisciplinary practices (IPs), and encourage links with formal and non-formal contexts. In methodological terms, the project comprises three phases (clarification, monitoring and dissemination) and is based on an essentially qualitative methodology. It is expected that the knowledge produced will provides the basis for the design of IPs.

Keywords: Interdisciplinarity; teacher training practices; higher education; international Cooperation

\section{Introdução}

As orientações das políticas educativas internacionais, tais como o Quadro Estratégico EF 2020 (CUE, 2009) e as Metas Educativas 2021 (OEI, 2010) definem prioridades que vão no sentido de melhorar a qualidade de educação e a formação inicial e continuada de professores; garantir as competências essenciais (EC, 2012); reforçar a investigação científica e promover oportunidades de educação ao longo da vida. Tais prioridades encaminham-se, necessariamente, para uma formação de professores de qualidade.

Sem negar a importância de muitas das mudanças iniciadas na formação inicial e no desenvolvimento profissional de professores e, inclusive, na sua capacidade transformadora, torna-se pertinente evidenciar algumas estratégias que contribuem para desenvolver novas competências e formas de ensinar e aprender, tais como as competências de planificar, implementar e avaliar PIs. Estas estratégias são assinaladas como entre as que melhor atendem as necessidades atuais dos estudantes do ensino superior (ES).

Tendo como contexto a formação de professores e considerando que nesta se contempla/inclui a dos educadores, este texto organiza-se em torno de/a:

(i) Significado(s) de PIs no ES

(ii) Relevância de PIs em contexto de formação de professores

(iii) Um projeto de identificação, caraterização e divulgação de PIs no ES - Projeto PRINT.

\section{Práticas interdisciplinares no ensino superior: seus significados}

Interdisciplinaridade não é um termo científico a que corresponda a uma definição única. Também PIs no ES estão (e estiveram sempre) imbuídas de múltiplos significados, perspetivas e juízos de valor. Pese embora a existência de variados significados, tais práticas estiveram sempre associadas a uma interação entre disciplinas "one in which two or more disciplines are brought together, preferably in such a way that the disciplines interact with one another and have some effect on one another's perspectives" (Rowntree, 1982, p. 135). De acordo com muitos autores, profissionais da educação e investigadores (ex. DEA/FBE, 2008; Pombo, 2004, 2006; Repko, 2008), é ponto assente que, em 
educação, tais práticas pressupõem "intensas relações conceituais estabelecidas entre as diferentes ciências e métodos de ensino cada vez mais problematizantes, estimuladores, desafiantes e dialéticos" (Thiesen, 2008, p. 552). Pressupõem também diferentes metodologias, ferramentas interpretativas e linguagens para analisar um problema central, assunto ou tema (Ivanitskaya, Clark, Montgomery \& Primeau, 2002).

Outros autores realçam que estas práticas têm no seu cerne a integração de diferentes perspetivas disciplinares, com o intuito de criar uma explicação ou propor uma solução para um assunto que seria inatingível através de meios disciplinares únicos (Miller \& Mansilla, 2004; Mansilla, Dan \& Middlebrooks, 2004). Estes autores consideram que a integração que ocorre não é um fim em si, mas um meio para atingir um objetivo, e que as disciplinas não são simplesmente justapostas: "disciplines are not simply juxtaposed but deeply intertwined - where the findings in one domain raise new questions in another, which in turn illuminate the problem at hand" (Miller \& Mansilla, 2004, p. 4).

Em torno destes pressupostos, assume-se que PIs na formação de professores, são práticas que se desenvolvem no âmbito de um curso de formação, vinculadas a um currículo e programas oficiais dos $1 .^{\circ} \mathrm{e}$ 2. ${ }^{\circ}$ ciclos de estudos.

Os responsáveis das diferentes unidades curriculares colaboram entre si, numa perspetiva integrada, que pode ir da (i) simples comunicação de ideias, à integração de (ii) conceitos, (iii) campos epistemológicos, (iv) terminologias, (v) metodologias de planificação, implementação e avaliação de práticas, (vi) procedimentos e (vii) organização da investigação e do ensino correspondentes.

Estas práticas, considerando a posição de Pombo (2006), podem subdividir-se em práticas de importação, de cruzamento, de descentração, de convergência e de comprometimento.

Entende-se por:

Práticas de importação, as que consistem "na "cooptação", a favor da disciplina "importadora", de "conceitos, métodos e instrumentos já provados noutras disciplinas" (Pombo, 2006, p. 231);

Práticas de cruzamento, as que consistem num "processo de fecundação recíproca das disciplinas envolvidas" (p. 232);

Práticas de descentração, as que "têm na sua origem a irrupção de problemas impossíveis de reduzir às disciplinas tradicionais" (...), "querendo-se com isto significar que não há propriamente uma disciplina que constitua o ponto de partida ou irradiação do problema, ou que seja o ponto de chegada do trabalho interdisciplinar. Há um policentrismo de disciplinas ao serviço do crescimento do conhecimento" [que pode dar origem] a "novas disciplinas" (p. 234);

Práticas de convergência, quando "a interdisciplinaridade passa, não tanto pela concertação prévia de uma metodologia, mas pelo convite à convergência de perspectivas em torno de um determinado objecto de análise (...) não implicando modificações estruturais nas disciplinas envolvidas ( $\mathrm{p}$. 233);
Práticas de comprometimento, quando resulta "de um esforço conjugado que visa, não apenas trocar informações ou confrontar métodos, mas fazer circular um saber, explorar activamente todas as suas possíveis complementaridades, explorar possibilidades de polinização cruzada (...), [com o objectivo de] encontrar "soluções técnicas para a resolução de problemas que resistem às contingências históricas em constante evolução" (p. 235).

Para a operacionalização de PIs, são necessários contextos facilitadores, quer a nível institucional (político-educativo), quer a nível curricular e pessoal (predisposição de cada um).

\section{Relevância de práticas interdisciplinares em contexto de formação de profissionais da educação}

Os professores, tal como os educadores, ex. de infância, desempenham um papel vital na sociedade, ao auxiliar os seus estudantes a desenvolverem o seu potencial de crescimento pessoal, social e de bem-estar. Desempenham ainda um papel relevante ao contribuírem para que os mesmos desenvolvam um leque complexo de competências de que irão precisar no exercício da sua atividade profissional (CE, 2017). Para além destas, os estudantes necessitam igualmente das que os capacitem para enfrentar os desafios humanísticos, científicos e tecnológicos.

De forma a auxiliá-los nessa tarefa, os professores, responsáveis pela formação de professores, necessitam de desenvolver novas estratégias. Tais estratégias passam pela promoção de PIs, por serem consideradas por muitos (ex. Jacob, 2015), as que melhor atendem às necessidades atuais dos estudantes do ES. Fundamentalmente, são estratégias que visam, essencialmente, contribuir para desenvolver competências e formas de ensinar e aprender, tais como as competências de planificar, implementar e avaliar PIs. Muitos estudos (DEA/FBE, 2008; Holley, 2009; Jacob, 2015) reforçam a importância destas práticas nas IES, como forma de os formandos (professores e educadores) explorarem, da melhor e mais ampla maneira possível, as suas competências académicas, e assim contribuírem face aos desafios atuais, para a melhoria da qualidade dos processos de ensino e de aprendizagem. Nessa linha, importa que as IES, ao promoverem a interdisciplinaridade, a articulem com uma formação sintonizada com a realidade social, numa perspetiva de aprendizagem ao longo da vida e a operacionalizem numa articulação efetiva entre a teoria e prática (OECD, 2011). Em suma, para promover PIs: "interdisciplinarity via enhanced collaboration between researchers from relevant academic fields, in particular interdisciplinary cooperation between natural and social sciences within a single institution or between institutions" (OECD, 2011) é crucial uma formação de professores de qualidade.

\section{O projeto PRINT}

Tendo como contexto: (i) as recentes recomendações da União Europeia (EU, 2016) para a melhoria na qualidade da educação em IES, nomeadamente as que apostam em estratégias de internacionalização ao nível da 
investigação; (ii) os desígnios gerais do Instituto Politécnico de Viseu (IPV, Portugal), de diversificar e incrementar a cooperação internacional, bem como (iii) estudos de referência (ex. European Commission/EACEA/Eurydice, 2015) que destacam a importância de se promoverem PIs em IES de formação de professores e de agentes educativos, concebeu-se um projeto de investigação, designado de PRINT, pelo seu manifesto interesse em se imprimirem PIs.

Trata-se de um projeto de cooperação internacional que contempla IES portuguesas, como a Escola Superior de Educação de Viseu (ESEV) e brasileiras, como o Centro de Educação à Distância da Universidade do Estado de Santa Catarina, cuja cooperação está sendo formalizada por meio de protocolo de intenções entre o IPV e a UDESC. Perfilha-se de que a cooperação internacional permita enriquecer o conhecimento sobre tais práticas. $\mathrm{O}$ projeto contempla ainda parcerias com instituições públicas de cariz cultural (ex. Museu Nacional Grão Vasco, Viseu, Portugal), como via enriquecedora da articulação interdisciplinar.

Atendendo a estes aspetos, uma equipa interdisciplinar na sua raiz, constituída por professores de diferentes áreas disciplinares da ESEV, em colaboração com professores do Centro de Educação à Distância da Universidade do Estado de Santa Catarina (CEADUDESC), leva a cabo, de 2017 a 2018, o projeto PRINT: Dimensões, princípios e objetivos de práticas interdisciplinares no ensino superior - um estudo no âmbito da cooperação entre o Brasil e Portugal.

Implementado desde janeiro de 2017, o projeto estrutura-se em três fases sequenciais (I, II e III) e tem como finalidade geral, identificar, caracterizar e partilhar, ao nível de IES, iniciativas desenvolvidas nos cursos de formação de professores, que potenciem a promoção de competências de planificação, implementação e avaliação de PIs, incluindo as que incentivem ligações com contextos formais e não formais.

Como objetivos, o projeto propõe-se:

1. Efetuar a clarificação concetual e terminológica de termos científicos envolvidos com interdisciplinaridade (enquadra a Fase I e a tarefa 1 que lhe subjaz);

2. Monitorizar as iniciativas de planificação, implementação e avaliação de PIs (enquadra a Fase II e as tarefas 2 a 4 que lhe subjazem);

3. Sintetizar, sistematizar e divulgar o conhecimento produzido através da criação de uma associação em rede de IES, organizada para o efeito (enquadra a Fase III e as tarefas 5 a 8 que lhe subjazem), conforme o Tabela 1.

Tabela 1.

Fases e tarefas do Projeto PRINT

\begin{tabular}{cl}
\hline Fases & \multicolumn{1}{c}{$\begin{array}{c}\text { Tarefas numeradas e respetivos } \\
\text { objetivos }\end{array}$} \\
\hline I- Clarificação & $\begin{array}{l}\text { 1. Clarificação terminológica e } \\
\text { concetual dos termos associados a PIs }\end{array}$
\end{tabular}

2. Mapeamento das IES que, nos cursos de formação de professores, desenvolvam PIs.

3. Produção de instrumentos de recolha

II- Monitorização de dados (IRD)

4. Implementação dos IRD

5. Tratamento de dados e análise de resultados. Elaboração de relatório intermédio

6. Conceção e planeamento de um seminário em Portugal

7. Realização do seminário

III- Síntese, 8. Elaboração do relatório final

Sistematização e 9. Publicações para divulgação dos Divulgação resultados do projeto

10. Criação de uma rede de IES para partilha, divulgação e reflexão sobre PIs

A clarificação (Fase I), pela sua relevância em qualquer fase inicial de um projeto de investigação (Brynard \& Hanekom, 1997; Nuopponen, 2010), é crucial, dado à partida, e fruto da própria composição da equipa, se reconhecer a multiplicidade de significados atribuídos à interdisciplinaridade e à necessidade de os clarificar.

Já a monitorização (Fase II), sendo um tipo particular de avaliação formativa (UNDP, 2009), é considerada necessária por permitir avaliar o estado de situação das PIs nas IES, e averiguar se estas estão a alcançar os resultados desejados com a sua implementação.

Em particular, com esta segunda fase, pretende-se:

1) listar as IES que, nos cursos de formação de professores, priorizem PIs formais em contextos formal e não formal;

2) caraterizar o papel das suas estruturas diretivas, em termos de orientações para o processo de planificação, implementação e avaliação de tais práticas;

3) descrever, analisar e refletir sobre as diferentes iniciativas de planificação, implementação e avaliação das PIs que estão a decorrer nas IES portuguesas e brasileiras.

Em relação à última fase - síntese, sistematização e divulgação do conhecimento produzido - pretende-se que a equipa conceba uma associação em rede de IES, onde se possa i) reunir e divulgar uma lista de boas PIs, ii) fomentar uma reflexão continuada sobre tais práticas e, iii) sugerir ideias para as melhorar e/ou as reformular nas situações que as requeiram.

Em termos metodológicos e dado que a escolha do método se deve fazer em função da natureza do problema a estudar (Bogdan \& Biklen, 1994), opta-se por um método misto, embora com pendor qualitativo, inscrito num paradigma construtivista e interpretativo (Creswell, 2003). No que concerne à recolha de dados, opta-se pela inquirição (por questionário, focus group e entrevista) aos participantes de IES de Portugal e Brasil - diretores ou coordenadores de curso, professores, investigadores, estudantes do $1 .^{\circ}$ e $2 .^{\circ}$ ciclo de estudos -, pela observação e por recolha documental (ver Quadro 2). O projeto prevê ainda inquirição a diretores e/ou técnicos de Instituições Públicas de Cariz Cultural (IPCC) que desenvolvam colaboração ao nível educativo com as IES participantes. 
O tratamento de dados implicará:

(1) Reunião e seleção/organização de dados para o seu tratamento (quantitativo ou qualitativo);

(2) Tratamento de dados: os de natureza quantitativa por estatística descritiva e os de natureza qualitativa por análise de conteúdo. Esta última é orientada por categorias que emergem dos objetivos de investigação e dos próprios dados (Bardin, 2004);

(3) Análise dos resultados obtidos, após a triangulação por tratamento estatístico e análise de conteúdo.

Este projeto envolve participantes de Portugal e do Brasil, ao nível de (i) IES: diretores ou coordenadores de curso, professores e estudantes de licenciatura e de mestrado, e ao nível de (ii) Instituições públicas de cariz cultural (IPCC): diretores e outros colaboradores/ técnicos que colaboram em iniciativas de cariz educativo.

No quadro seguinte (Tabela 2) sintetizam-se os diversos participantes e técnicas e instrumentos de recolha de dados para cada uma das fases do projeto.

Tabela 2.

Objetivos, participantes e técnicas de recolha de dados no âmbito do projeto PRINT

\begin{tabular}{|c|c|c|}
\hline $\begin{array}{c}\text { Fases } \\
\text { do } \\
\text { projet } \\
\text { o } \\
\end{array}$ & Participantes & $\begin{array}{l}\text { Técnicas/instrumento } \\
\mathrm{s}\end{array}$ \\
\hline $\mathrm{I}$ & Equipa do projeto PRINT & Recolha documental \\
\hline II & $\begin{array}{c}\text { Equipa do Projeto PRINT } \\
\text { Diretores/coordenadores, } \\
\text { Professores e estudantes de } \\
\text { IES } \\
\text { Diretores e técnicos de } \\
\text { IPCC }\end{array}$ & $\begin{array}{c}\text { Inquirição por } \\
\text { questionário } \\
\text { Inquirição por focus } \\
\text { group } \\
\text { Inquirição por } \\
\text { entrevista }\end{array}$ \\
\hline
\end{tabular}

Como principais produtos prevê-se, então:

i) Instrumentos de recolha de dados;

ii) Publicações em revistas;

iii) Comunicações em encontros científicos;

iv) Seminário de divulgação de resultados

v) Relatórios (dois).

vi) Espaço no Moodle do Instituto Politécnico de Viseu, de partilha, divulgação e reflexão sobre PIs no ES.

Com este estudo, espera-se ficar em condições de poder reunir e partilhar PIs em contexto de cooperação nacional e internacional, e reforçar as relações entre investigadores, os implementadores de tais práticas e demais interessados.

\section{Considerações finais}

Reconhecendo que muitos dos desafios humanísticos, científicos e tecnológicos têm quase sempre caráter interdisciplinar e somente podem ser resolvidos ou solucionados por equipas de profissionais de diversas áreas, o projeto que se apresenta constitui uma oportunidade para reunir e partilhar entre equipas de dois países, PIs desenvolvidas no âmbito da formação de professores.

Espera-se que o conhecimento produzido na consecução das suas fases sirva de background e estímulo a outros projetos de cariz interdisciplinar, para definição de políticas de formação e capacitação de professores em geral, assim como para melhorar a qualidade dos processos de ensino e aprendizagem.

Por último, projetos como o apresentado vêm reforçar a importância de se aliar a investigação à formação e educação, como forma de reajustar e/ou inovar práticas no ES.

\section{Referências}

Bardin, L. (2004). Análise de Conteúdo. $3{ }^{\text {ed }}$ Lisboa: Edições 70.

Bogdan, R. \& Biklen, S. (1994). Investigação Qualitativa em Educação - uma introdução à teoria e aos métodos. Porto: Porto Editora.

Brynard P. \& Hanekom, S. X. (1997). Introduction to research in Public Administration and related academic disciplines. Pretoria: Van Schaik Academic Publishers.

CE [Comissão Europeia] (2007). Comunicação da Comissão ao Conselho e ao Parlamento Europeu. Melhorar a qualidade da formação académica e profissional dos docentes. http://ftp.infoeuropa.eurocid.pt/files/database/0000390 01-000040000/000039820.pdf

Creswell, J. W. (2003). Research Design: qualitative, quantitative and mixed methods approaches. 2. ed. California: Sage Publications Inc.

CUE [Conselho da União Europeia] (2009). Informações oriundas das instituições e dos órgãos da união europeia. Conclusões do Conselho de 12 de Maio de 2009 sobre um quadro estratégico para a cooperação no domínio da educação e da formação - EF 2020.

DEA/FBE (Danish Business Research AcademyDanmarks Erhvervsforsknings Akademi/Danish Forum for Business Education) (2008). - Thinking across disciplines - interdisciplinarity in research and education. Copenhagen: http://www.ucm.es/info/eurohum/docs/dea.pdf

EC [European Commission] (2012). Communication from the Commission to the European parliament, the council, the European Economic and Social Committee and the Committee of the regions - Rethinking Education:Investing in skills for better socio-economic Strasbourg: EC 20.11.2012, COM (2012) 669.

EC/EACEA/Eurydice [European Commission/ Education, Audiovisual and Culture Executive Agency/ Education and Youth Policy Analysis] (2015). The Teaching Profession in Europe: Practices, Perceptions, and policies. Eurydice Report. Luxembourg: Publications Office of the European Union. http:/eacea.ec.europa.eu/education/eurydice/documen ts/thematic_reports/184EN.pdf

Holley, K. A. (2009). Understanding Interdisciplinary Challenges and Opportunities in Higher Education: ASHE Higher Education Report, Volume 35, Number 2, $1^{\text {st }} \quad$ Edition. Hoboken, $\quad$ NJ: http://onlinelibrary.wiley.com/doi/10.1002/aehe.3502/ epdf

Ivanitskaya, L., Clark, D., Montgomery, G., \& Primeau, R. (2002). Interdisciplinary learning: Process and 
outcomes. Innovative Higher Education. 27 (2), 95111.

Jacob, J. (2015). Interdisciplinary trends in higher education. Palgrave Communications. 1(15001), 1-5. http://www.palgrave-

journals.com/articles/palcomms20151

Mansilla, V. B., Dan D., \& Middlebrooks, K. (2004). Building Bridges Across Disciplines: Organizational and Individual Qualities of Exemplary Interdisciplinary Work. Interdisciplinary Studies Project, Project Zero, Harvard Graduate School of Education. GoodWork Paper 16. Cambridge, MA.

Miller, M., \& Mansilla, V. B. (2004). Thinking across perspectives and disciplines. GoodWork Project Report Series, Number 27. Cambridge, MA.

Nuopponen, A. (2010). Methods of concept analysis - a comparative study. Part 1 of 3. LSP Journal Language for special purposes, professional communication, knowledge management and cognition, 1 (2), 4-12. http://lsp.cbs.dk

OECD [Organisation for Economic Co-operation and Development] (2011). Global Science Forum - Report on Opportunities, Challenges and Good Practices in International Research Cooperation between Developed and Developing Countries. April 2011 https://www.oecd.org/sti/sci-tech/47737209.pdf

OEI [Organização dos Estados Iberoamericanos] (2010). Metas educativas 2021. La educación que queremos para la generación de los bicentenários. Madrid: Cudipal. http://www.oei.es/metas2021/libro.htm

Pombo, O. (2004). Interdisciplinaridade: Ambições e limites (pp. 73-104). Lisboa: Relógio d'Água.

Pombo, O. (2006). Práticas interdisciplinares. Sociologias, Porto Alegre, 8(15): 208-249.

Repko, A. F. (2008). Interdisciplinary Research: Process and Theory. Thousand Oaks, CA: SAGE Publications, Inc.

Rowntree, D. (1982). A dictionary of education. Totowa, NJ: Barnes \& Noble Books.

Thiesen, J. S. (2008). A interdisciplinaridade como um movimento articulador no processo ensinoaprendizagem. Revista Brasileira de Educação. 13(39), 545-598.

UNDP [UnitedNationsDevelopmentProgramme] (2009). Handbook on Planning, Monitoring and Evaluating for Development results. New York: UNDP. http://web.undp.org/evaluation/evaluations/handbook/ english/documents/pme-handbook.pdf

\section{Agradecimentos}

Projeto financiado pelo Instituto Politécnico de Viseu (IPV)/Caixa Geral de Depósitos (CGD) (Referência do projeto: PROJ/CI\&DETS/CGD/0003) 